\title{
AN EXTREMAL PROBLEM FOR POLYNOMIALS WITH NONNEGATIVE COEFFICIENTS
}

\author{
GRADIMIR V. MILOVANOVIĆ
}

\begin{abstract}
Let $W_{n}$ be the set of all algebraic polynomials of exact degree $n$ whose coefficients are all nonnegative. For the norm in $L^{2}[0, \infty)$ with generalized Laguerre weight function $w(x)=x^{\alpha} e^{-x}(\alpha>-1)$, the extremal problem $C_{n}(\alpha)=$ $\sup _{P \in w_{n}}\left(\left\|P^{\prime}\right\| /\|P\|\right)^{2}$ is solved, which completes a result of A. K. Varma [7].
\end{abstract}

1. In this paper we give the complete solution of a problem which has been investigated recently by $\mathrm{A}$. K. Varma (see $[7,8])$. This problem is related to some previous integral inequalities of Varma $[9,10]$ and also to the classical inequalities of A. Markov [4], P. Erdös [1], G. G. Lorentz [2, 3], G. Szegö [5], and P. Turan [6].

Let $W_{n}$ be the set of all algebraic polynomials of exact degree $n$, all coefficients of which are nonnegative, i.e.,

$$
W_{n}=\left\{P_{n} \mid P_{n}(x)=\sum_{k=0}^{n} a_{k} x^{k}, a_{k} \geqq 0(k=0,1, \ldots, n)\right\} .
$$

We denote by $W_{n}^{0}$ the subset of $W_{n}$ for which $a_{0}=0$ (i.e., $P_{n}(0)=0$ ).

Let $w(x)=x^{\alpha} e^{-x}(\alpha>-1)$ be a weight function on $[0, \infty)$, and let $\|f\|^{2}=(f, f)$, where

$$
(f, g)=\int_{0}^{\infty} w(x) f(x) g(x) d x \quad\left(f, g \in L^{2}[0, \infty)\right)
$$

In [7] Varma has investigated the problem of determining the best constant in the inequality

$$
\left\|P_{n}^{\prime}\right\|^{2} \leqq C_{n}(\alpha)\left\|P_{n}\right\|^{2}
$$

where $P_{n} \in W_{n}$. In fact, he has proved

THEOREM A. Let $P_{n}(x)$ be an algebraic polynomial of exact degree $n$ with nonegative coefficients. Then for $\alpha \geqq(\sqrt{5}-1) / 2$,

$$
\int_{0}^{\infty}\left(P_{n}^{\prime}(x)\right)^{2} x^{\alpha} e^{-x} d x \leqq \frac{n^{2}}{(2 n+\alpha)(2 n+\alpha-1)} \int_{0}^{\infty} P_{n}^{2}(x) x^{\alpha} e^{-x} d x,
$$

equality holding for $P_{n}(x)=x^{n}$. For $0 \leqq \alpha \leqq 1 / 2$ we have

$$
\int_{0}^{\infty}\left(P_{n}^{\prime}(x)\right)^{2} x^{\alpha} e^{-x} d x \leqq \frac{1}{(2+\alpha)(1+\alpha)} \int_{0}^{\infty} P_{n}^{2}(x) x^{\alpha} e^{-x} d x
$$

Received by the editors March 29, 1984.

1980 Mathematics Subject Classification. Primary 26C05, 41 A44. 
Moreover, (1.2) is also best possible in the sense that for $P_{n}(x)=x^{n}+\lambda x$ the expression on the left can be made arbitrarily close to the expression on the right by choosing $\lambda$ positive and sufficiently large.

The case $\alpha=1$ was considered in [8]. The cases $\alpha \in(-1,0)$ and $\alpha \in(1 / 2$, $(\sqrt{5}-1) / 2)$ are still open.

2. The object of this paper is to determine

$$
C_{n}(\alpha)=\sup _{P_{n} \in W_{n}} \frac{\left\|P_{n}^{\prime}\right\|^{2}}{\left\|P_{n}\right\|^{2}}
$$

for all $\alpha \in(-1, \infty)$ and, thus, to give a complete solution of the extremal problem (1.1). Note that the supremum in (2.1) is attained for some $P_{n} \in W_{n}^{0}$. Indeed,

$$
\sup _{P_{n} \in W_{n}} \frac{\left\|P_{n}^{\prime}\right\|}{\left\|P_{n}\right\|}=\sup _{\substack{P_{n} \in W_{n}^{0} \\ a_{0} \geqq 0}} \frac{\left\|P_{n}^{\prime}\right\|}{\left\|P_{n}+a_{0}\right\|}=\sup _{P_{n} \in W_{n}^{0}} \frac{\left\|P_{n}^{\prime}\right\|}{\left\|P_{n}\right\|} .
$$

We begin by proving three lemmas:

LEMMA 1. If $P_{n} \in W_{n}$ then for every $x \geqq 0$ the inequality

$$
x\left(P_{n}^{\prime}(x)^{2}-P_{n}(x) P_{n}^{\prime \prime}(x)\right) \leqq P_{n}^{\prime}(x) P_{n}(x)
$$

holds.

Proof. Let $P_{n} \in W_{n}$, i.e., $P_{n}(x)=\sum_{k=0}^{n} a_{k} x^{k}$ with $a_{k} \geqq 0(k=0,1, \ldots, n)$. Using the Cauchy-Schwarz inequality

$$
\left|\sum_{k=0}^{n} x_{k} y_{k}\right|^{2} \leqq\left(\sum_{k=0}^{n}\left|x_{k}\right|^{2}\right)\left(\sum_{k=0}^{n}\left|y_{k}\right|^{2}\right)
$$

for $x_{k}=a_{k}^{1 / 2} x^{k / 2}$ and $y_{k}=k a_{k}^{1 / 2} x^{k / 2}(x \geqq 0)$, we obtain

$$
\left(\sum_{k=0}^{n} k a_{k} x^{k}\right) \leqq\left(\sum_{k=0}^{n} a_{k} x^{k}\right)\left(\sum_{k=0}^{n} k^{2} a_{k} x^{k}\right),
$$

which is equivalent to (2.2).

LEMMA 2. If $P_{n} \in W_{n}^{0}$, then for the integrals

$$
\begin{aligned}
J_{n}(\alpha) & =\int_{0}^{\infty} x^{\alpha} e^{-x} P_{n}^{\prime}(x)^{2} d x \\
I_{n, i}(\alpha) & =\int_{0}^{\infty} x^{\alpha} e^{-x} P_{n}(x) P_{n}^{(i)}(x) d x \quad(i=0,1,2)
\end{aligned}
$$

the following recurrence relations hold:

$$
\begin{aligned}
I_{n, 2}(\alpha) & =I_{n, 1}(\alpha)-\alpha I_{n, 1}(\alpha-1)-J_{n}(\alpha) \quad(\alpha>-1), \\
2 I_{n, 1}(\alpha) & =I_{n, 0}(\alpha)-\alpha I_{n, 0}(\alpha-1) \quad(\alpha>-2) .
\end{aligned}
$$

The proof of this lemma is a simple application of integration by parts and will be omitted. We note that the integrals $I_{n, 1}(\alpha)$ and $I_{n, 0}(\alpha-1)$ exist for $\alpha>-2$ because $P_{n}(0)=0$. 
From Lemmas 1 and 2 there immediately follows

LEMMA 3. If $P_{n} \in W_{n}^{0}$, then for $\alpha>-1$,

$$
J_{n}(\alpha) \leqq \frac{1}{4}\left\{I_{n, 0}(\alpha)+(1-2 \alpha) I_{n, 0}(\alpha-1)+(\alpha-1)^{2} I_{n, 0}(\alpha-2)\right\} .
$$

THEOREM. The best constant $C_{n}(\alpha)$ defined in (2.1) is

$$
C_{n}(\alpha)= \begin{cases}1 /(2+\alpha)(1+\alpha) & \left(-1<\alpha \leqq \alpha_{n}\right) \\ n^{2} /(2 n+\alpha)(2 n+\alpha-1) & \left(\alpha_{n} \leqq \alpha<+\infty\right)\end{cases}
$$

where

$$
\alpha_{n}=\frac{1}{2}(n+1)^{-1}\left(\left(17 n^{2}+2 n+1\right)^{1 / 2}-3 n+1\right) .
$$

Proof. Let $P_{n} \in W_{n}^{0}$, i.e., $P_{n}(x)=\sum_{k=1}^{n} a_{k} x^{k}\left(a_{k} \geqq 0\right)$. Then

$$
P_{n}(x)^{2}=\sum_{k=2}^{2 n} b_{k} x^{k} \quad\left(b_{k} \geqq 0\right)
$$

and

$$
\left\|P_{n}\right\|^{2}=I_{n, 0}(\alpha)=\sum_{k=2}^{2 n} b_{k} \Gamma(k+\alpha+1),
$$

where $\Gamma$ is the gamma function. Using Lemma 3 we obtain

$$
4 J_{n}(\alpha) \leqq \sum_{k=2}^{2 n} b_{k}\left\{\Gamma(k+\alpha+1)+(1-2 \alpha) \Gamma(k+\alpha)+(\alpha-1)^{2} \Gamma(k+\alpha-1)\right\},
$$

i.e.,

$$
J_{n}(\alpha) \leqq \sum_{k=2}^{2 n} H_{k}(\alpha) b_{k} \Gamma(k+\alpha+1)
$$

where

$$
H_{k}(\alpha)=\frac{1}{4} \cdot k^{2} /(k+\alpha)(k+\alpha-1) .
$$

From (2.5) it follows that

$$
\left\|P_{n}^{\prime}\right\|^{2} \leqq\left(\max _{2 \leqq k \leqq 2 n} H_{k}(\alpha)\right)\left\|P_{n}\right\|^{2}
$$

so

$$
C_{n}(\alpha) \leqq \max _{2 \leqq k \leqq 2 n} H_{k}(\alpha) \text {. }
$$

Determining the maximum of $f(x)=x^{2} /(x+\alpha)(x+\alpha-1)$ on the interval $[2,2 n]$, we find that

$$
\max _{2 \leqq k \leqq 2 n} H_{k}(\alpha)= \begin{cases}H_{2}(\alpha) & \text { if }-1<\alpha \leqq \alpha_{n}, \\ H_{2 n}(\alpha) & \text { if } \alpha_{n} \leqq \alpha<+\infty,\end{cases}
$$

where $\alpha_{n}$ is given by (2.4). 
In order to show that $C_{n}(\alpha)$ defined in (2.3) is best possible, i.e. that $C_{n}(\alpha)=$ $\max _{2 \leqq k \leqq 2 n} H_{k}(\alpha)$, we consider $\tilde{P}_{n}(x)=x^{n}+\lambda x(\lambda \geqq 0)$ and set

$$
Q_{n}(\lambda)=\left\|\tilde{P}_{n}^{\prime}\right\|^{2} /\left\|\tilde{P}_{n}\right\|^{2}
$$

By a simple computation we find that

$$
Q_{n}(\lambda)=\frac{n^{2} \Gamma(2 n+\alpha-1)+2 \lambda n \Gamma(n+\alpha)+\lambda^{2} \Gamma(\alpha+1)}{\Gamma(2 n+\alpha+1)+2 \lambda \Gamma(n+\alpha+2)+\lambda^{2} \Gamma(\alpha+3)} .
$$

Since

$$
Q_{n}(0)=n^{2} /(2 n+\alpha)(2 n+\alpha-1)=H_{2 n}(\alpha)
$$

and

$$
\lim _{\lambda \rightarrow \infty} Q_{n}(\lambda)=1 /(\alpha+1)(\alpha+2)=H_{2}(\alpha),
$$

we conclude that $\tilde{P}_{n}(x)=x^{n}$ is an extremal polynomial for $\alpha \geqq \alpha_{n}$. Furthermore, if $-1<\alpha \leqq \alpha_{n}$, there exists a sequence of polynomials, for example, $p_{n, k}(x)=x^{n}+k x$, $k=1,2, \ldots$, for which

$$
\lim _{k \rightarrow \infty} \frac{\left\|p_{n, k}^{\prime}\right\|^{2}}{\left\|p_{n, k}\right\|^{2}}=C_{n}(\alpha)
$$

REMARK. From (2.4) we have $\alpha_{1}=(\sqrt{5}-1) / 2, \quad \alpha_{2}=(\sqrt{73}-5) / 6, \alpha_{3}=$ $(\sqrt{10}-2) / 2$, etc. The sequence $\left(\alpha_{k}\right)$ is decreasing, i.e., $\alpha_{1}>\alpha_{2}>\alpha_{3}>\cdots>\alpha_{\infty}$, where $\alpha_{\infty}=\lim _{n \rightarrow \infty} \alpha_{n}=(\sqrt{17}-3) / 2 \cong 0.56155$.

Acknowledgment. The author is grateful to Professor W. Gautschi for his careful reading of the paper and useful suggestions for better and more complete formulations of the material.

\section{REFERENCES}

1. P. Erdös, Extremal properties of derivatives of polynomials, Ann. of Math. (2) 41 (1940), 310-313.

2. G. G. Lorentz, The degree of approximation by polynomials with positive coefficients, Math. Ann. 151 (1963), 239-251.

, Derivatives of polynomials with positive coefficients, J. Approx. Theory 1 (1968), 1-4.

4. A. A. Markov, On a problem of D. I. Mendeleev, Izv. Akad. Nauk SSSR Ser. Mat. 62 (1889), 1-24.

5. G. Szegö, On some problems of approximations, Magyar Tud. Akad. Mat. Kutato Int. Dozl. 2 (1964), $3-9$.

6. P. Turan, Remarks on a theorem of Erhard Schmidt, Mathematica (2) 25 (1960), 373-378.

7. A. K. Varma, Derivatives of polynomials with positive coefficients, Proc. Amer. Math. Soc. 83 (1981), 107-112.

8. Some inequalities of algebraic polynomials having real zeros, Proc. Amer. Math. Soc. 75 (1979), 243-250.

9. An analogue of some inequalities of P. Turan concerning algebraic polynomials having all zeros inside [-1, + 1], Proc. Amer. Math. Soc. 55 (1976), 305-309.

10. An analogue of some inequalities of $P$. Turan concerning algebraic polynomials having all zeros inside [-1, +1]. II, Proc. Amer. Math. Soc. 69 (1978), 25-33.

Faculty of Electronic Engineering, Department of Mathematics, University of Nis, BeOgRADSKa 14, P. O. Box 7318000 Nis, YugoslaVia 